(W)

Check for

updates

Cite as

Nano-Micro Lett.

(2020) $12: 52$

Received: 3 December 2019

Accepted: 1 February 2020

Published online: 17 February 2020

(C) The Author(s) 2020

\section{Hierarchical Self-assembly of Well-Defined Louver-Like P-Doped Carbon Nitride Nanowire Arrays with Highly Efficient Hydrogen Evolution}

\author{
Bo $\mathrm{Li}^{1}$, Yuan $\mathrm{Si}^{1}$, Qian Fang ${ }^{1}$, Ying $\mathrm{Shi}^{2}$, Wei-Qing Huang ${ }^{1}$, Wangyu $\mathrm{Hu}^{1}{ }^{凶}$, \\ Anlian Pan ${ }^{1}$, Xiaoxing Fan ${ }^{3}$, Gui-Fang Huang ${ }^{1} \bowtie$ \\ $\triangle$ Wei-Qing Huang, wqhuang@hnu.edu.cn; Wangyu Hu, wyuhu@hnu.edu.cn; Gui-Fang Huang, \\ gfhuang@hnu.edu.cn \\ 1 Department of Applied Physics, College of Physics and Electronics, and College of Materials Science \\ and Engineering, and State Key Laboratory of Chemo/Biosensing and Chemometrics, College of Chemistry \\ and Chemical Engineering, Hunan University, Changsha 410082, People's Republic of China \\ 2 Department of Physics and Tsinghua-Foxconn Nanotechnology Research Center, Tsinghua University, \\ Beijing 100084, People's Republic of China \\ 3 College of Physics, Liaoning University, Shenyang 110036, People's Republic of China
}

\title{
HIGHLIGHTS
}

- The self-assembled louver-like P-doped carbon nitride (L-PCN) nanowires is firstly constructed.

- L-PCN nanowires show efficient charge transport, as demonstrated by experimental and theoretical approach.

- L-PCN nanowires exhibit significantly boosted HER activity of $1,872.9 \mu \mathrm{mol} \mathrm{h}^{-1} \mathrm{~g}^{-1}(\lambda>420 \mathrm{~nm})$.

\begin{abstract}
Self-assembled nanostructure arrays integrating the advantages of the intrinsic characters of nanostructure as well as the array stability are appealing in advanced materials. However, the precise bottom-up synthesis of nanostructure arrays without templates or substrates is quite challenging because of the general occurrence of homogeneous nucleation and the difficult manipulation of noncovalent interactions. Herein, we first report the precisely manipulated synthesis of well-defined louver-like P-doped carbon nitride nanowire arrays (L-PCN) via a supramolecular self-assembly method by regulating the

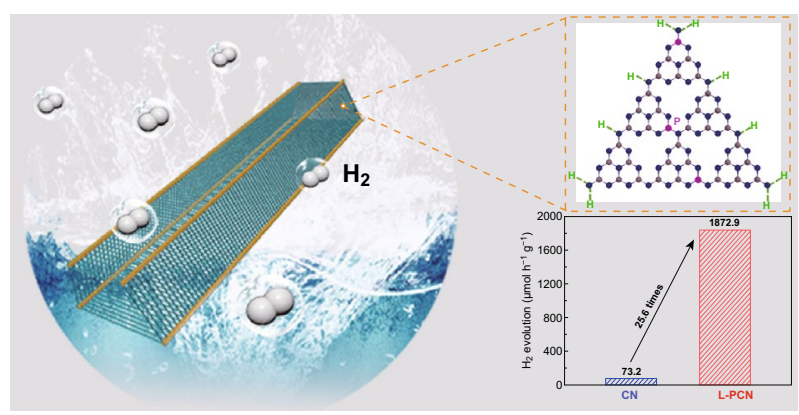
noncovalent interactions through hydrogen bond. With this strategy, $\mathrm{CN}$ nanowires align in the outer frame with the separation and spatial location achieving ultrastability and outstanding photoelectricity properties. Significantly, this self-assembly L-PCN exhibits a superior visible light-driven hydrogen evolution activity of $1872.9 \mu \mathrm{mol} \mathrm{h}^{-1} \mathrm{~g}^{-1}$, rendering a $\sim 25.6$-fold enhancement compared to bulk $\mathrm{CN}$, and high photostability. Moreover, an apparent quantum efficiency of $6.93 \%$ is achieved for hydrogen evolution at $420 \pm 15 \mathrm{~nm}$. The experimental results and first-principles calculations demonstrate that the remarkable enhancement of photocatalytic activity of L-PCN can be attributed to the synergetic effect of structural topology and dopant. These findings suggest that we are able to design particular hierarchical nanostructures with desirable performance using hydrogen-bond engineering.
\end{abstract}

KEYWORDS Self-assembly; Carbon nitride; P-doped; Nanowire arrays; Hydrogen evolution 


\section{Introduction}

The controlled self-assembly has become a key technology in the development of diversified bottom-up nanostructured materials with desirable properties for numerous applications ranging in electronics, optoelectronic devices to catalysis [1-5]. Recently, research focused on self-assembled nanostructures, such as nanowires [6-8], nanopillars [9], nanotubes [10-13], and nanosheets [14], has led to substantial advances. Major efforts in this field are so far emphasized on the single nanostructure to elucidate structure-property relationships. However, self-assembly of single nanostructure into welldefined nanostructure arrays without templates or substrates has met with little success so far. The reason for the reluctance of researchers to enter this field is given by the difficulties encountered in the design and precise control of the diverse weak noncovalent or covalent bonds interactions, involving in $\pi-\pi$ and charge transfer interactions, hydrogen/halogen bonding, and van der Walls force in the self-assembly process $[8$, $11,15]$. Even with templates or substrates assisted, the growth of nanostructured arrays is still extremely limited by the selfassembly directions (vertical growth) $[4,6,16]$. Therefore, developing a feasible pathway to releasing the potential of single nanostructures self-assembly into diversified nanostructure arrays is still a great challenge.

Polymeric graphitic carbon nitride $(\mathrm{CN})$ has been becoming a rising star material in recent years due to its extraordinary features and potential applications in the areas of hydrogen evolution, water oxidation, and artificial photosynthesis [17-20]. However, bulk $\mathrm{CN}$ obtained by the direct thermal polymerization process generally presents low surface area and fast charge recombination, which significantly restricts its applications [21-23]. To improve the performance of $\mathrm{CN}$, various strategies have been developed and the promising pathways are mainly classified into two categories. One way is the development of various $\mathrm{CN}$ nanostructures with optimized physicochemical and optical properties $[24,25]$. The other is heteroatom doping to optimizing the electronic and bandgap structures [26-30]. Particularly, much attention has been paid to the self-assembledspecific supramolecular $\mathrm{CN}$ nanostructures because of their convenient operation and template-free properties in the synthesis process [10, 31-34]. More exhilaratingly, polymeric $\mathrm{CN}$ is generally synthesized via thermal polycondensation of carbon, nitrogen, and hydrogen containing precursors (such as melamine and urea), giving a promising direction of supermolecular self-assembly $\mathrm{CN}$ nanostructures based on hydrogen bonding, where the chaotic molecules can be self-assembled because hydrogen bonding has strong direction and saturation [35-39]. Unfortunately, although various $\mathrm{CN}$ micro-/nanostructures have been fabricated through thermal polycondensation of the hydrogen containing precursors, the single nanostructures such as microtubes and nanotubes are generally accompanied with serious aggregation and restacking during the practical applications, leading to poor recycling performance [10, 40-42]. Further achievement of hierarchical self-assembly of single CN nanostructure into well-defined nanostructure arrays to impart the $\mathrm{CN}$-based materials with ultrastability as well as highly efficient performance has not yet been reported.

In this work, we first report an approach for the hierarchical self-assembly of well-defined louver-like P-doped CN nanowire arrays $(\mathrm{L}-\mathrm{PCN})$ whereby $\mathrm{CN}$ nanowires are aligned in the outer frame with the separation and spatial location to retard restacking and improve the hydrogen evolution performance of $\mathrm{CN}$. The hierarchical self-assembly strategy based on the hydrogen bonding interaction between melamine and cyanuric acid develops a precisely well-defined supramolecular precursor via kinetically controlled growth pathway (Fig. S1) under the stirring solution. Moreover, the facile modulation in the formation ratio of the hydrogen bonds by adding appropriate amount of phosphoric acid, which promotes the hydrolysis of melamine to cyanic acid, can realize the quantitative manipulation of the assembly clearance spaces and the exposed degree of active sites in L-PCN. Significantly, the as-prepared L-PCN exhibits a reputably hydrogen evolution activity $\left(1872.9 \mu \mathrm{mol} \mathrm{h}^{-1} \mathrm{~g}^{-1}\right)$ under visible light irradiation and renders a $\sim 25.6$-fold enhancement compared to bulk $\mathrm{CN}$. Moreover, an apparent quantum efficiency (AQY) of $6.93 \%$ at $420 \pm 15 \mathrm{~nm}$ is achieved for L-PCN. Characterization of the morphologies as well as specific surface area, charge carrier separation, and transfer efficiency measurements reveals that this outstanding hydrogen evolution performance is attributed to the synergistic effect toward the hierarchical self-assembly louver-like $\mathrm{CN}$ nanowire arrays and P-doping.

\section{Experimental}

\subsection{Preparation of L-PCN Nanowire Arrays}

First, melamine (10 mmol) with different amounts of phosphoric acid $(0.5,1.0,1.5$, and $2.0 \mathrm{~mL}$, the corresponding 
$\mathrm{pH}$ value is $3.5,2.5,2$, and 1.5 ) and cyanuric acid (10 mmol) were, respectively, dissolved in deionized water $(100 \mathrm{~mL})$ with continuous stirring for $20 \mathrm{~min}$; after melamine and cyanuric acid were completely dissolved, then the melamine solution was slowly poured into the cyanuric acid solution. The mixture solution was centrifuged, washed, and dried at $60{ }^{\circ} \mathrm{C}$. Finally, the resultant solids were heated at $550{ }^{\circ} \mathrm{C}$ for $2 \mathrm{~h}$. The catalyst obtained by adding $0.5,1.0,1.5$, and $2.0 \mathrm{~mL}$ of phosphorous acid was named as L-PCN-0.5, L-PCN-1.0, L-PCN-1.5, and L-PCN-2.0, respectively.

\subsection{Preparation of Bulk Graphitic CN}

Bulk graphitic carbon nitride $(\mathrm{CN})$ was prepared by similar process of L-PCN but without adding phosphorous acid.

\subsection{Characterization}

The texture properties of the as-prepared samples were examined by X-ray diffraction (XRD, Siemens D-5000 diffractometer) with $\mathrm{Cu} K \alpha$ irradiation source $(\lambda=0.154 \mathrm{~nm})$. Fourier-transform infrared (FT-IR) spectra were recorded on a IR Affnity-1 FTIR spectrometer using $\mathrm{KBr}$ pellet as the background. The chemical state of $\mathrm{C}, \mathrm{N}$, and $\mathrm{P}$ elements was measured through $\mathrm{X}$-ray photoelectron spectroscopy (XPS, PHI Quantera X-ray photoelectron spectrometer) with $300 \mathrm{~W}$ Al K $\alpha$ radiation. Scanning electron microscope (SEM, Hitachi S-4800) and transmission electron microscope (TEM, FEI Tecai F20) were used to observe the morphology of samples. The specific surface areas of the samples were calculated using the Brunauer-Emmett-Teller (BET) method. UV-Vis diffuse reflectance spectra were recorded on a UV-2450 UV-Vis spectrophotometer (Shimadzu Systems, Japan). The photoluminescence (PL) spectra were recorded on a F-2500 fluorescence spectrometer with pulsed xenon discharge lamps upon excitation by incident light of $350 \mathrm{~nm}$ at room temperature. Time-resolved fluorescence decay spectra were collected using a streak camera (C10910, Hamamatsu), in which the emission signal was reflected on the streak camera by a mirror.

\subsection{Photocatalytic Test}

The hydrogen evolution activity was carried out in a Pyrex top-irradiation reaction vessel connected to a closed glass gas system (LabSolar-III AG, Perfectlight Limited, Beijing). A $300 \mathrm{~W}$ xenon lamp with a cutoff filter $(\lambda \geq 420 \mathrm{~nm})$ as light source. Generally, photocatalyst (50 mg) loaded with $\approx 1 \% \mathrm{Pt}$ and methyl alcohol (10 vol\%) as sacrificial electron donor were dispersed in aqueous solution $(300 \mathrm{~mL})$. The reactant solution was evacuated several times before irradiation to remove the air prior. The temperature of the reaction solution was maintained at $10{ }^{\circ} \mathrm{C}$ by a flow of cooling water. The evolved gases were analyzed by gas chromatography (GC7900, Techcomp) equipped with a thermal conductive detector (TCD) for quantification and a 5 A molecular sieve column, using nitrogen as the carrier gas.

The apparent quantum yield (AQY) was measured using a similar experimental setup, only with designated monochromic light $(420 \mathrm{~nm})$ to perform the hydrogen evolution. The photointensity was averaged at $20 \mathrm{~mW} \mathrm{~cm}^{-2}$ representative points by PLS-SXE300D photoradiometer, and the irradiation area was approximately $16 \mathrm{~cm}^{2}$. The AQY was estimated based on Eq. 1:

$\mathrm{AQY}=\frac{\text { number of evolved } \mathrm{H}_{2} \text { molecules } \times 2}{\text { number of incident photons }} \times 100$

Photocatalytic degrading organic pollutants of rhodamine $\mathrm{B}\left(\mathrm{RhB}, 10 \mathrm{mg} \mathrm{L}^{-1}\right)$ and bisphenol A (BPA, $\left.10 \mathrm{mg} \mathrm{L}^{-1}\right)$ were measured under visible light irradiation (300 W halogen lamp). Typically, $10 \mathrm{mg}$ of catalysts was added into $40 \mathrm{~mL}$ model pollutant solution. The suspensions were magnetically stirred in the dark to obtain absorption-desorption equilibrium, and $4 \mathrm{~mL}$ of the suspensions was collected at irradiation time intervals and then centrifuged to remove the photocatalyst particles. The concentrations of $\mathrm{RhB}$ and BPA were, respectively, analyzed by measuring the absorbance of supernatant at 664 and $275 \mathrm{~nm}$ on a TU-1910 UV-Vis spectrophotometer.

\subsection{Photoelectrochemical Test}

Photoelectrochemical measurements were conducted in a conventional three-electrode cell system using Chen Hua 
electrochemical station (Shanghai). The fluorine-doped tin oxide (FTO) transparent conductive film glass deposited with samples, Pt wire, and $\mathrm{Ag} / \mathrm{AgCl}$ electrode were, respectively, used as working electrodes, counterelectrode, and reference electrode. 0.2 $\mathrm{M} \mathrm{Na}_{2} \mathrm{SO}_{4}$ aqueous solution $(\mathrm{pH}=6.8)$ was used as the electrolyte. For Mott-Schottky plots, various frequencies of obtained samples were, respectively, at 1.0, 2.3, and $3.1 \mathrm{kHz}$. For Nyquist plot measurements, the frequency was ranged from $10^{-2}$ to $10^{6} \mathrm{~Hz}$. The photocurrent response of the photocatalysts as light on and off was measured without bias voltage. Besides, linear sweep voltammetry (LSV) plots for the obtained samples were studied in $0.5 \mathrm{M} \mathrm{H}_{2} \mathrm{SO}_{4}$ aqueous solution with a scan rate of $10 \mathrm{mV} \mathrm{s}^{-1}$.

\subsection{DFT Calculations}

All our calculations are based on density functional theory (DFT) in conjunction with the projector-augmented wave (PAW) potential as implemented by the Vienna ab initio Simulation Package (VASP) [43, 44]. The Perdew-Burke-Ernzerhof generalized gradient approximation (GGA) with the approach of DFT-D2 correction is adopted to correct the weak van der Waals-like interactions $[45,46]$. As is known, the GGA functional usually underestimates the band gap of semiconductors. Therefore, the screened hybrid Heyd-Scuseria-Ernzerhof 2006 (HSE06) functional has been employed to obtain accurate electronic structures and optical properties $[47,48]$. The cutoff energy for the plane-wave basis set is $500 \mathrm{eV}$. Geometry optimization is carried out before single-point energy calculation, and the force on the atoms is less than $0.01 \mathrm{eV} \AA^{-1}$. The first Brillouin zone is sampled with a Monkhorst-Pack grid of $5 \times 5 \times 1$. All atomic positions were fully relaxed until the force is less than $0.01 \mathrm{eV} \AA^{-1}$. We set the criterion for the total energy to be $1.0 \times 10^{-6} \mathrm{eV}$. A vacuum space of $15 \AA$ along the $z$ direction is used to avoid artificial interaction.

\section{Results and Discussion}

The fabrication process of the hierarchical self-assembly of well-defined louver-like P-doped $\mathrm{CN}$ nanowire arrays (L-PCN) is shown in Fig. 1. As melamine, cyanuric acid, and phosphorous acid are dissolved in deionized water, phosphoric acid molecules are wrapped in or adsorbed on the immediately formed melamine-cyanuric acid (M-CA) micromolecule assembly, yielding a quadrangular-like P-containing supramolecular precursor. Typical SEM image in Fig. 2a reveals that the obtained quadrangularlike structure has a smooth surface and an edge length of 50-250 nm, indicating the successful molecule selfassembly between melamine and cyanuric acid. After pyrolysis, $\mathrm{P}$ atoms squeeze into the $\mathrm{CN}$ skeleton and the self-assembled louver-like $\mathrm{P}$-doped $\mathrm{CN}$ nanowire arrays (L-PCN) is formed (Fig. 2b, c); the yield of the synthesis is about $10 \%$. The transformation from quadrangularlike precursor to L-PCN nanowire arrays can be mainly ascribed to the release of various gases during pyrolysis, including $\mathrm{NH}_{3}, \mathrm{NO} / \mathrm{NO}_{2}, \mathrm{CO}_{2}$, and $\mathrm{PH}_{3}$. It is worth noting that the thermal polymerization of pure $\mathrm{M}-\mathrm{CA}$ supramolecular precursor (Fig. S2a) only results in irregular bulk CN structure (Fig. S3a). The reason for the distinctly difference morphology between bulk $\mathrm{CN}$ and L-PCN lies in the use of phosphoric acid in the reaction solution. The hierarchical self-assembly process based on the hydrogen bonding interaction toward melamine and cyanuric acid can be precisely controlled with phosphorous acid-assisted strategy, since some melamine molecules could be hydrolyzed into cyanuric acid in the presence of phosphorous acid, realizing the quantitative manipulation of the formation of hydrogen bonds. Simultaneously, the phosphorous acid molecules adsorbed on the inside and surface of the quadrangular-like precursor can release a large amount of gas during pyrolysis, which facilitates the separation and spatial location of the self-assembled $\mathrm{CN}$ nanowire arrays (Fig. S4). Different volumes of phosphoric acid added from 0.5 to $2.0 \mathrm{~mL}$ also lead to smooth quadrangular-like precursors (Fig. S2b-d) and louver-like nanowire arrays (Fig. S3b-d). Moreover, the clearance spaces among CN nanowires in L-PCN change with the increasing in phosphoric acid added, certifying the critical role of phosphoric acid in regulating the hydrogen bonds ratio and the final texture. The hierarchical self-assembly of louver-like nanowire arrays is also clearly observed in TEM images (Fig. 2d, e), and the hierarchical self-assembly of louver-like nanowire arrays with adequate open clearance spaces offers large active sites exposed, enhanced light harvesting, high charge carriers, and mass transport rate. In addition, the elemental mapping illustrated in Fig. $2 \mathrm{f}-\mathrm{i}$ demonstrates that $\mathrm{C}, \mathrm{N}$, and $\mathrm{P}$ are distributed uniformly on the surface of L-PCN-1.0 arrays. 




The mixed solution

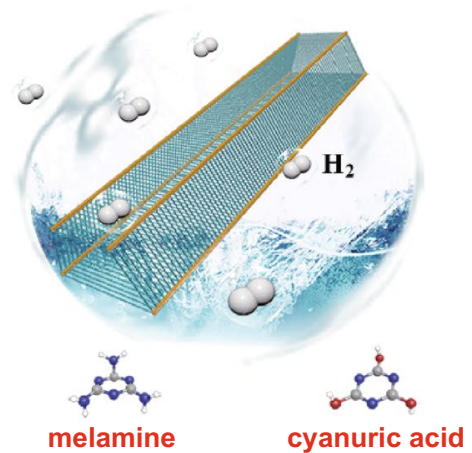

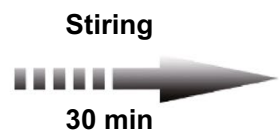
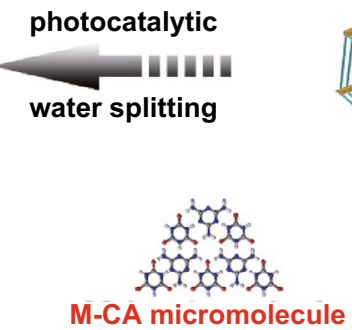
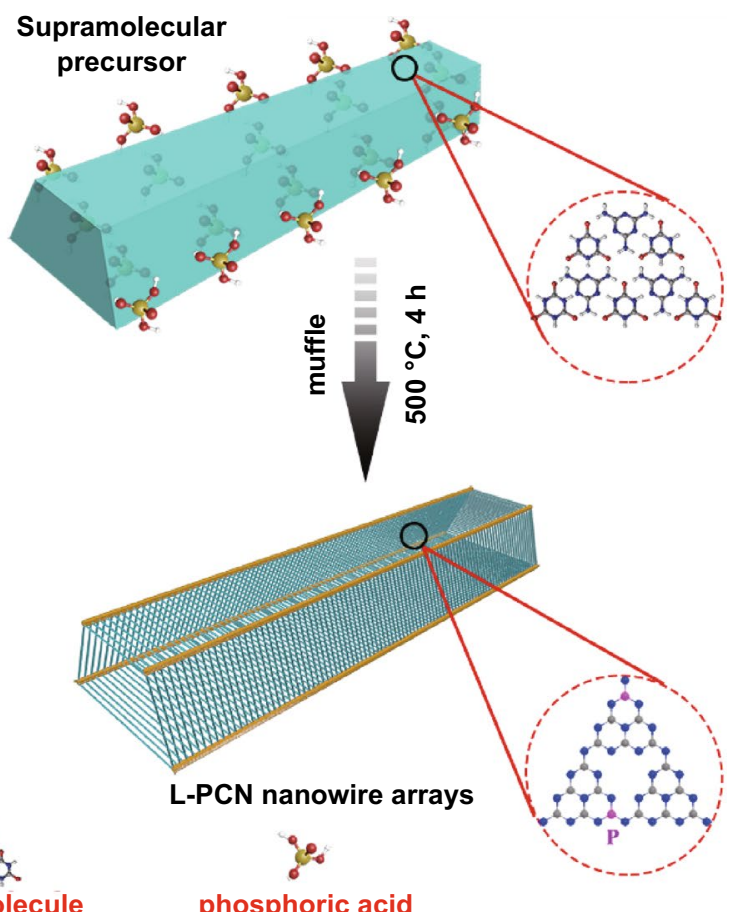

Fig. 1 Preparation process of L-PCN nanowire arrays. The quadrangular-like hydrogen-bonded supermolecular precursor is easily prepared by molecular cooperative assembly. Meanwhile, phosphoric acid molecules will be wrapped in or adsorbed on the precursors. After annealing, hierarchical self-assembly of CN nanowire arrays with well-defined louver-like nanostructures (L-CN) is eventually generated through releasing various gas, such as $\mathrm{NH}_{3}, \mathrm{NO} / \mathrm{NO}_{2}$, and $\mathrm{PH}_{3}$, and the $\mathrm{P}$ atoms are successfully doped in the internal framework

The unique hierarchical self-assembly of louver-like $\mathrm{CN}$ nanowire arrays motivates us to further study its Brunauer-Emmett-Teller (BET) surface area and pore volume. Thus, $\mathrm{N}_{2}$ adsorption-desorption isotherms of bulk $\mathrm{CN}$ and L-PCN are investigated. The typical IV adsorption isotherm is obviously observed in Fig. 3a, indicating the characteristic adsorption-desorption hysteresis with well-defined mesopore distribution. The BET surface area of L-PCN-1.0 is calculated to be $121 \mathrm{~m}^{2} \mathrm{~g}^{-1}$ from the linear part of the multipoint plot, which is approximately four times higher than that of bulk CN (ca. $33 \mathrm{~m}^{2} \mathrm{~g}^{-1}$ ). Compared to bulk $\mathrm{CN}$, the extremely high adsorption capacity in the high relative pressure $\left(\mathrm{P} / \mathrm{P}_{0}\right.$ from 0.8 to 1.0$)$ is clearly observed, suggesting the presence of abundant mesopores and/or macropores for L-PCN-1.0 [49, 50], which is well consistent with the SEM and TEM observation. In addition, L-PCN-1.0 also possess larger pore volume $\left(0.80 \mathrm{~cm}^{3} \mathrm{~g}^{-1}\right)$ compared to bulk $\mathrm{CN}\left(\right.$ ca. $\left.0.14 \mathrm{~cm}^{3} \mathrm{~g}^{-1}\right)$. The increased BET surface area and larger pore volume for L-PCN-0.5, L-PCN-1.5, and L-PCN2.0 are also achieved (Fig. S5 and Table S1) and further confirm that the hierarchical self-assembly of louver-like $\mathrm{CN}$ nanowire arrays can effectively promote the kinetics of catalytic reaction by increasing exposed active sites and facilitating mass transfer [51].

The texture properties and chemical structures of pristine $\mathrm{CN}$ and L-PCN are characterized by XRD patterns and FTIR spectroscopy. As shown in Fig. 3c, the XRD pattern for L-PCN-1.0 exhibits two characteristic peaks located at $13.2^{\circ}(100)$ and $27.4^{\circ}(002)$, which are, respectively, associated with in-plane structural packing motif and periodic stacking of layers along the $c$-axis in $\mathrm{CN}$ structure $[25,52]$. However, it is worth mentioning that the peak at $13.2^{\circ}$ and $27.4^{\circ}$ is sharply weakened compared to bulk $\mathrm{CN}$, revealing the broken intralayer long-range atomic order and the tris-triazine units are stacked in a perpendicular direction to the sheets via $\pi-\pi$ interactions [10]. With increasing phosphoric acid addition, the obtained L-PCN shows a gradually weaker diffraction peaks at $13.2^{\circ}$ (Fig. S6a), suggesting the smaller in-planar layer size owing to the "gas etching effect" in L-PCN during thermal condensation. Moreover, the peak at $27.4^{\circ}$ slightly shifts to lower $2 \theta$ degree (Fig. S6b), indicating that $\mathrm{P}$ atoms are successfully incorporated into the $\mathrm{L}-\mathrm{CN}$ framework [53]. For FT-IR spectrum in Fig. 3d, it is clear that L-PCN-1.0 possesses similar characteristic vibration 




Fig. 2 Structural characterization of L-PCN. a Typical SEM images of L-PCN-1.0 supramolecular precursor. b SEM images of L-PCN-1.0 after thermal treatment with $500{ }^{\circ} \mathrm{C}, 4 \mathrm{~h}$. $\mathbf{c}$ Quadrangular and louver model of the supramolecular precursor and L-PCN nanowire arrays. d, e TEM images of L-PCN-1.0. f-i EDS elemental mapping images of L-PCN-1.0

modes compared to bulk $\mathrm{CN}$, indicating that the P-doped hierarchical self-assembly of louver-like nanostructure array does not destroy the fundamental chemical structure of $\mathrm{CN}$. Nevertheless, a pianissimo weak band at $950 \mathrm{~cm}^{-1}$ attributed to the $\mathrm{P}-\mathrm{N}$ stretching mode is found for L-PCN-1.0 [28] and the stretching vibrations band at $808 \mathrm{~cm}^{-1}$ for bulk $\mathrm{CN}$ shifts to a higher wavenumber of $812 \mathrm{~cm}^{-1}$ for L-PCN-1.0 (Fig. S7a), further suggesting that $\mathrm{P}$ atoms are successfully incorporated into the triazine ring, which is in agreement with the XRD analysis. Particularly, compared to bulk CN, the gradually stronger band at $3000-3500 \mathrm{~cm}^{-1}$ (Fig. S7b) is associated with the stretching vibrations of $\mathrm{N}-\mathrm{H}$ bond in the marginal amino groups reconfirming the higher hydrogenbond ratio in the self-assembled L-PCN nanowire arrays.

XPS measurements are used to ascertain the surface chemical compositions and chemical state of $\mathrm{C}, \mathrm{N}$, and
$\mathrm{P}$ elements in L-PCN. The spectrum in Fig. S8 indicates that bulk $\mathrm{CN}$ is mainly composed of $\mathrm{C}, \mathrm{N}$, and $\mathrm{O}$ elements, while small amount of P can be detected for L-PCN-1.0, suggesting that $\mathrm{P}$ atoms are successfully incorporated into the $\mathrm{CN}$ framework. The high ratio of $\mathrm{C}$ element in $\mathrm{CN}$ and L-PCN-1.0 spectrum may be ascribed to the externally contaminated carbon sources, while $\mathrm{O}$ element may be attributed to the surface absorbed oxygen-containing species in the samples $[49,54]$. The C $1 s$ spectra of L-PCN1.0 can be fitted into three contributions according to the Gaussian rule and, respectively, located at 284.8, 286.4, and $288.1 \mathrm{eV}$, as shown in Fig. 4a. The peak in $288.1 \mathrm{eV}$ is attributed to $s p^{2}$-hybridized carbon atom bonds to the aliphatic amine in the aromatic rings $(\mathrm{N}-\mathrm{C}=\mathrm{N})$, and the peak at 284.8 and $286.4 \mathrm{eV}$ is, respectively, ascribed to the pure graphitic species $(\mathrm{C}-\mathrm{C})$ and $\mathrm{C}-\mathrm{NH}_{x}(x=1,2)$ on the edges 



Fig. 3 Texture properties and chemical structures of CN and L-PCN. a Nitrogen adsorption-desorption isotherms and b corresponding pore size distribution curves of $\mathrm{CN}$ and L-PCN-1.0. $\mathbf{c}, \mathbf{d}$ XRD patterns and FTIR spectra of CN and L-PCN-1.0

of heptazine units [55]. In particular, the ratio of $\mathrm{C}-\mathrm{NH}_{x} / \mathrm{C}$ increases from $28.51 \%$ for bulk $\mathrm{CN}$ to $28.58 \%$ for $\mathrm{L}-\mathrm{PCN}$ 1.0 (Table S2), indicating more hydrogen bonds in L-PCN1.0. The $\mathrm{N} 1 s$ spectrum for L-PCN-1.0 can be deconvoluted into four peaks at 398.6, 399.9, 401.1, and $404.1 \mathrm{eV}$, respectively (Fig. 4b). The peak located at $398.6 \mathrm{eV}$ is corresponded to the $s p^{2}$-hybridized aromatic in triazine rings [56, 57], whereas the peak located at $399.9 \mathrm{eV}$ is ascribed to the bridging $\mathrm{N}$ atoms in $\mathrm{N}_{3} \mathrm{C}$ groups, and the slight shift to higher binding energy compared to $\mathrm{CN}(399.6 \mathrm{eV})$ may be ascribed to the changed electron structure in L-PCN-1.0 (Fig. 4b and Table S3) [56]. The peak at $401.1 \mathrm{eV}$ is indexed to the amino functions carrying hydrogen $(\mathrm{C}-\mathrm{N}-\mathrm{H})$, and the weakness peak in the $\mathrm{N} 1 s$ spectra centered at $404.2 \mathrm{eV}$ is most probably attributed to the positive charge localization in heterocycles $[21,49,58]$. The peak ratio of the amino functions carrying hydrogen is found to be higher than that of bulk CN (Table S2), implying more hydrogen-bond ratio exists in L-PCN-1.0 and in good agreement with the change of $\mathrm{C} 1 s(286.4 \mathrm{eV})$. The survey spectrum of $\mathrm{P} 2 p$ signal is located at around $133.3 \mathrm{eV}$ (Fig. 4c), and the binding energy is higher than $\mathrm{P}-\mathrm{C}$ bonds $(131.5-132.5 \mathrm{eV})$ and lower than $\mathrm{P}=\mathrm{N}$ bonds, suggesting that $\mathrm{P}$ atoms most probably replace $\mathrm{C}$ in triazine rings of $\mathrm{CN}$ to form $\mathrm{P}-\mathrm{N}$ bonds (Fig. 4d) [28, 59-61], consistent with the FTIR spectra results (Fig. 3d). On the other hand, the surface atomic ratio of $\mathrm{P}$ element for L-PCN-1.0 is calculated about $0.25 \%$ (Table S4). Correspondingly, EDS test demonstrates that the $\mathrm{P}$ atomic content is $0.28 \%$ (Table S5), indicating the low ratio of $\mathrm{P}$ atoms in the $\mathrm{CN}$ nanowire arrays matrix.

The combination of $\mathrm{P}$ atom and unique self-assembled L-CN nanowire arrays would alter the optical properties and electronic band structure, which are studied by a combined analysis of optical absorption spectra and fluorescence emission spectra. The UV-Vis diffuse reflectance spectra (DRS) in Fig. 5a show that the absorption edge of L-PCN-1.0 shifts to 



Fig. 4 Surface chemical compositions and chemical states characterization of C, N, and P elements for CN and L-PCN. a-c High resolution of $\mathrm{C} 1 s, \mathrm{~N} 1 s$, and P $2 p$ XPS spectra of CN and L-PCN-1.0. d Proposed structure of L-PCN (carbon, nitrogen, and phosphorus atoms are, respectively, indicated by gray, blue, and pink spheres in the atomic model). (Color figure online)

longer wavelengths compared to bulk $\mathrm{CN}$, indicating that hierarchical self-assembly of $\mathrm{CN}$ nanowire arrays can effectively improve the visible light harvesting. Accordingly, the derived electronic band gaps from the Tauc plots reveal the narrowed bandgap energy of L-PCN-1.0 (2.71 eV) compared to $2.85 \mathrm{eV}$ for bulk CN (Fig. 5b) [62]. In addition, the band gaps for L-PCN-0.5, L-PCN-1.5, and L-PCN-2.0 are 2.79, 2.74, and $2.76 \mathrm{eV}$, respectively (Fig. S9) and further confirm the positive role of $\mathrm{P}$ integration and self-assembled L-CN nanowire arrays in enhancing the visible light harvesting ability.

The steady-state photoluminescence (PL) emission spectra of samples are shown in Figs. 5c and S10. Compared to bulk CN, all L-PCN catalysts show lower PL intensity, indicating that hierarchical self-assembled louver-like $\mathrm{CN}$ nanowires can efficiently suppress the recombination of photogenerated electron-holes. Moreover, the emission peak red-shifts from about $440 \mathrm{~nm}$ for bulk CN to around $480 \mathrm{~nm}$ for L-PCN-1.0, which is consistent with DRS spectra. The time-resolved fluorescence decay spectra are also studied to get quantitative information of the photogenerated charge carriers, as shown in Fig. 5d. All spectra are monitored at the wavelength of the maximum emission peak of each product. The recorded emission decay data are analyzed and fitted with a biexponential kinetics model (Eq. 2) [63]:

$I(t)=A_{1} \mathrm{e}^{-t / \tau_{1}}+A_{2} \mathrm{e}^{-t / \tau_{2}}$

which generates two lifetime values, a fast $\tau_{1}$ and slow $\tau_{2}$ decay component, and the corresponding amplitudes, $A_{1}$ and $A_{2}$. The average PL lifetime ( $\tau$ ) is deduced by Eq. 3 [60]:

$(\tau)=\frac{A_{1} \tau_{1}^{2}+A_{2} \tau_{2}^{2}}{A_{1} \tau_{1}+A_{2} \tau_{2}}$

The derived two components of the lifetime and their relative amplitudes are given in Table S5. The resulting average PL lifetime of L-PCN-1.0 (1.66 ns) is 4.04 times longer than the pristine $\mathrm{CN}(0.41 \mathrm{~ns})$. Specifically, L-PCN-1.0 shows the 

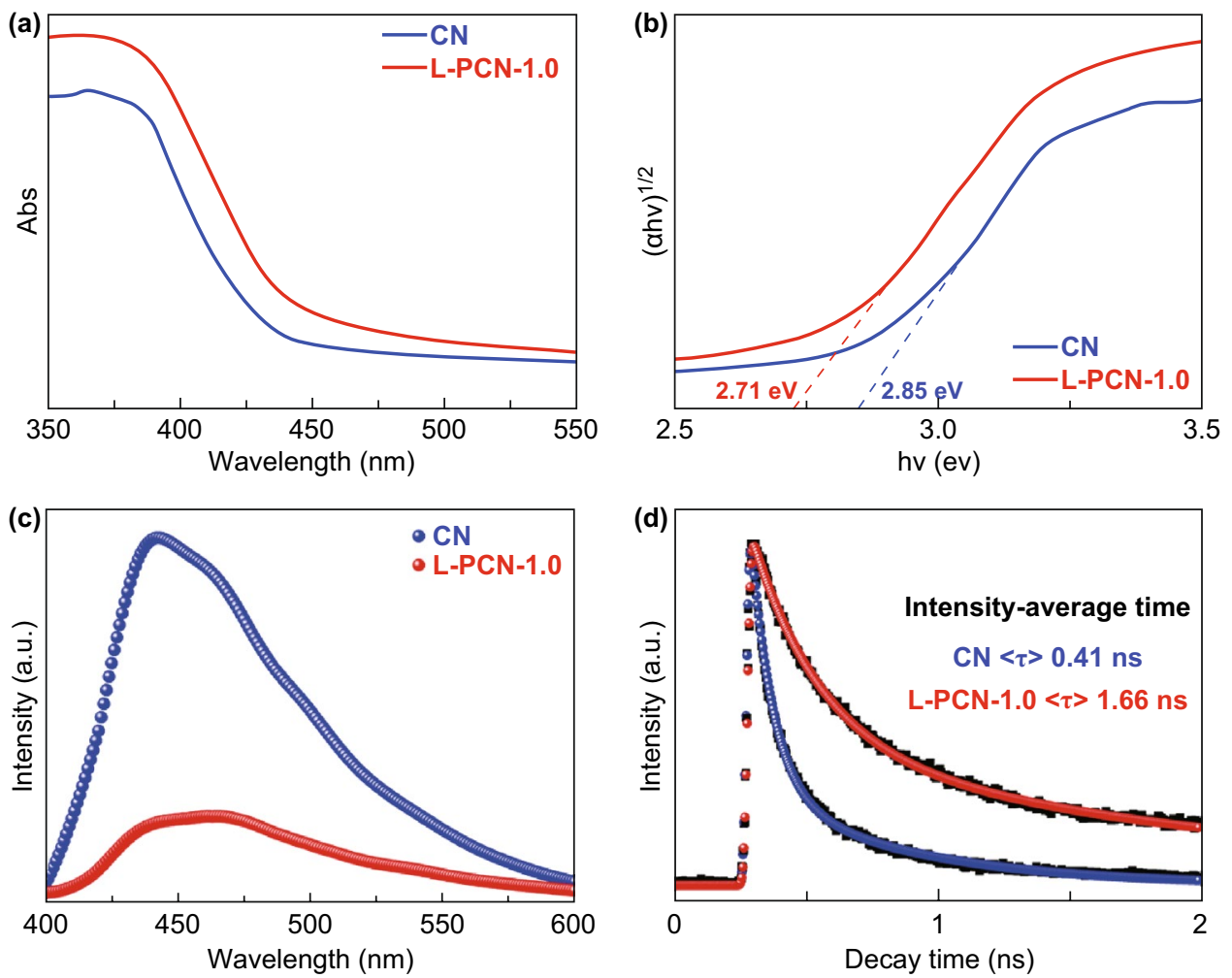

Fig. 5 Optical properties characterization of CN and L-PCN. a UV-Vis DRS and b corresponding band gaps of CN and L-PCN-1.0 determined through the Tauc plots transformed from Kubelka-Munk function. c Photoluminescence emission spectra (with $350 \mathrm{~nm}$ excitation wavelength at room temperature) of $\mathrm{CN}$ and L-PCN-1.0. d Time-resolved photoluminescence decay spectra of CN and L-PCN-1.0. These spectra were recorded with the excitation of $400 \mathrm{~nm}$ from a picosecond pulsed light-emitting diode at room temperature

fast (0.26 ns) for a majority of charge carriers $(62.18 \%)$ and slow (1.95 ns) with contributions of $37.82 \%$, respectively, while bulk $\mathrm{CN}$ exhibits the shortest lifetime $(0.08 \mathrm{~ns})$ for a large percentage $(75.85 \%)$ of charge carriers, suggesting the significantly different emission pathway. In addition, the decreased PL intensity and increased average PL lifetime of L-PCN-0.5, L-PCN-1.5, and L-PCN-2.0 (Figs. S10, S11 and Table S6) have also been achieved, suggesting that the constructed louver-like $\mathrm{CN}$ nanowires with $\mathrm{P}$ doping are synergistically lengthened the lifetimes of charge carriers.

To make sure that the as-prepared L-PCN catalyst is suitable for photocatalytic hydrogen evolution under visible light, the electronic band structure is determined by the analysis of Mott-Schottky plots at different frequencies of 1.0, 2.3, and $3.1 \mathrm{kHz}$, respectively (Figs. 6a and $\mathrm{S} 12)$. The positive slope of $\mathrm{CN}$ and $\mathrm{L}-\mathrm{PCN}-1.0$ originates from the typical $n$-type characteristic semiconductor, and the flat band potential is ascertained as $x$-intercept in Mott-Schottky plots [24, 50]. In addition, the smaller slope of L-PCN-1.0 in Mott-Schottky plot reflects a higher electron donor density, which is very helpful for improving photocatalytic performance owing to the increased electrical conductivity [10, 28], possibly caused by the unique nanostructure and P-doping effect. The flat band potentials of $\mathrm{CN}$ and L-PCN-1.0 are determined to be about -0.76 and $-1.21 \mathrm{~V}$ with the reversible hydrogen electrode (RHE). Compared to bulk CN, the $0.45 \mathrm{~V}$ upshift potential for L-PCN-1.0 will lead to a large thermodynamic driving force in photocatalytic hydrogen production. Generally, the flat band potential can be used to approximately estimate the CB position $[64,65]$. Herein, combined with the bandgap energy (Fig. 5b), the band structure of the as-prepared photocatalysts can also be obtained (Fig. 6b). Both CN and L-PCN-1.0 satisfied the thermodynamic conditions for photocatalytic water splitting.

Nyquist curve of electrochemical impedance spectroscopy (EIS) and transient photocurrent responses are used to investigate the charge transport behavior of $\mathrm{CN}$ and L-PCN catalysts. Generally, the smaller radius of the Nyquist plots corresponds to the faster electron transfer kinetics of the 

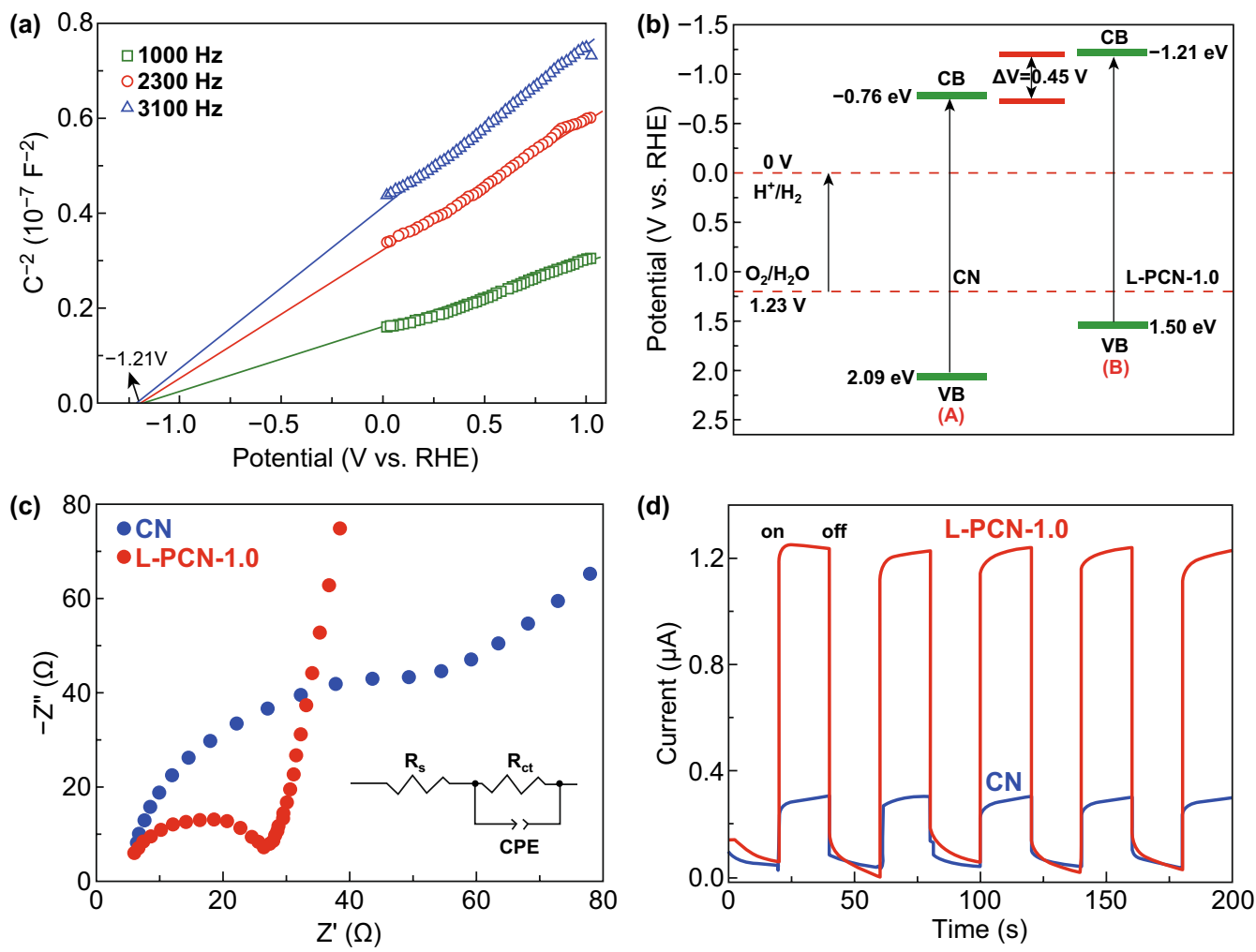

Fig. 6 Photoelectrochemical characterization of CN and L-PCN. a Mott-Schottky plots with various frequencies of 1.0, 2.3, and 3.1 kHz for L-PCN-1.0 in $0.2 \mathrm{M} \mathrm{Na}_{2} \mathrm{SO}_{4}$ aqueous solution $(\mathrm{PH}=6.8)$. b Band structure diagram for $\mathrm{CN}$ and L-PCN-1.0. c Electrochemical impedance spectroscopy (EIS) Nyquist plots for CN and L-PCN-1.0 obtained in the dark. d Transient photocurrents of CN and L-PCN-1.0 in $0.2 \mathrm{M} \mathrm{Na}_{2} \mathrm{SO}_{4}$ aqueous solution

redox reaction and lower charge transfer resistance $\left(R_{\mathrm{ct}}\right)$ [66]. The remarkable decrease in Nyquist plot diameter for L-PCN-1.0 (Fig. 6c), L-PCN-0.5, L-PCN-1.5, and L-PCN2.0 (Fig. S13) catalysts reflects the smaller charge transfer resistance from electrode to electrolyte molecules, originating from the better conductivity of L-PCN, which is in good agreement with the Mott-Schottky tests. Beyond that, transient photocurrent response of $\mathrm{CN}$ and L-PCN under visible light illumination $(\lambda>420 \mathrm{~nm})$ in Figs. $6 \mathrm{~d}$ and $\mathrm{S} 14$ shows that all samples exhibited a sensitive photocurrent response during five times on/off irradiation cycles under visible light. Particularly, L-PCN-1.0 shows the largest photocurrent value of about $1.3 \mu \mathrm{A} \mathrm{cm}^{-2}$, which is 4.33 times of bulk $\mathrm{CN}\left(0.3 \mu \mathrm{A} \mathrm{cm}^{-2}\right)$, suggesting a high-efficiency charge separation ability for L-PCN-1.0. In addition, the photocurrent responses do not decay with increase in illuminated time, indicating that L-PCN-1.0 catalyst provides a stable quantity of electrons and holes during irradiation.
The photocatalytic activity of $\mathrm{CN}$ and L-PCN catalysts is evaluated via hydrogen production from water splitting under visible light irradiation $(\lambda>420 \mathrm{~nm})$. As shown in Fig. 7a, $10.994 \mu \mathrm{mol}$ for bulk $\mathrm{CN}$ and $280.935 \mu \mathrm{mol} \mathrm{H}_{2}$ for L-PCN-1.0 are generated after $3 \mathrm{~h}$ visible light irradiation (methyl alcohol as the sacrificial agent and all samples are loading $1 \% \mathrm{Pt}$ ), respectively. Besides that, the hydrogen evolution amount of L-PCN-0.5 (44.94 $\mu \mathrm{mol}), \mathrm{L}-\mathrm{PCN}-1.5$ $(108.53 \mu \mathrm{mol})$, and L-PCN-2.0 (100.65 $\mu \mathrm{mol})$ samples is also higher than that of over bulk CN (Fig. S15), arising from their relatively high surface area, enhanced visible light absorption, and high charge transport capability (Table S1, Figs. S9 and S13). The average hydrogen production rate (Fig. 7b) for L-PCN-1.0 can be calculated to be $1872.9 \mu \mathrm{mol} \mathrm{h}^{-1} \mathrm{~g}^{-1}$, which is 25.6 times higher than that of bulk $\mathrm{CN}\left(73.2 \mu \mathrm{mol} \mathrm{h} \mathrm{h}^{-1} \mathrm{~g}^{-1}\right)$. The apparent quantum yield (AQY) for hydrogen evolution of L-PCN-1.0 under monochromatic light irradiation conditions is estimated to be 



Fig. 7 Photo-/electrocatalytic activity characterization of $\mathrm{CN}$ and L-PCN. a Photocatalytic activity test of $\mathrm{H}_{2}$ evolution performance of $\mathrm{CN}$ and L-PCN-1.0 with $1 \mathrm{wt} \% \mathrm{Pt}$ under visible light irradiation $(\lambda>420 \mathrm{~nm}) . \mathbf{b} \mathrm{H}_{2}$ evolution rate of $\mathrm{CN}$ and L-PCN-1.0. $\mathbf{c}$ Four cycling test of photocatalytic $\mathrm{H}_{2}$ evolution of L-PCN-1.0. d Linear sweep voltammetry (LSV) plots of CN and L-PCN-1.0 catalysts for $\mathrm{HER}$ catalysis in $0.5 \mathrm{M} \mathrm{H}_{2} \mathrm{SO}_{4}$

about $6.93 \%$ at $420 \pm 15 \mathrm{~nm}$. It is deserved to be mentioned that the catalytic performance of L-PCN-1.0 is better than most reported values of P-doped CN (Table S7). Better yet, L-PCN-1.0 is reused over four cycles without noticeable deactivation of its hydrogen production (Fig. 7c), indicating the high stability of photocatalytic hydrogen yielding, which is in good agreement with the photocurrent results (Fig. 5d). Besides that, the electrocatalytic activity for hydrogen evolution in $0.5 \mathrm{M} \mathrm{H}_{2} \mathrm{SO}_{4}$ medium over bulk $\mathrm{CN}$ and L-PCN1.0 cathodes is investigated in a typical three-electrode cell with reference to the RHE. Obviously, L-PCN-1.0 catalyst shows higher electrocatalytic activity for hydrogen evolution in contrast to bulk $\mathrm{CN}$ and other L-PCN with different phosphorus acid added (Fig. S16). Moreover, L-PCN-1.0 also possesses excellent photocatalytic activity to degrade organic pollutants (RhB and BPA) under visible light irradiation (Fig. S17).

To elucidate the fundamental effects of $\mathrm{P}$ doping in $\mathrm{CN}$, we performed DFT calculations. According to the experiment results (Fig. 4), the $\mathrm{P}$ atoms are introduced in the computational model, in which the $\mathrm{P}$ atom replaces the corner carbon atoms (Fig. S18). The density of states projected on each atomic species of pristine $\mathrm{CN}$ and $\mathrm{P}$-doped $\mathrm{CN}$ is displayed in Fig. 8. The valence band maximum (VBM) of $\mathrm{CN}$ is mainly consisted of $\mathrm{N}$ orbital, while the conduction band minimum (CBM) is contributed by the $\mathrm{C}$ and $\mathrm{N}$ orbital together (Fig. 8a). After doping P atom, the interfacial formation energy increases slightly to $12.22 \mathrm{meV} \AA^{-1}$ (Fig. S19) compared to pure CN (11.76 $\left.\mathrm{meV}^{-1}\right)$. As the interlayer distance increases, the interfacial formation energy is also lower than that before doping, suggesting a stronger interlayer binding in P-doped $\mathrm{CN}$. After doping $\mathrm{P}$ atoms into the $\mathrm{CN}$, the bandgap decreases from 3.05 to $2.88 \mathrm{eV}$ (Fig. 8b), consistent with the general trend seen in the experiment data above. The $\mathrm{P}$ doping does not alter the composition of the band edges, but induces a new mid-gap state above the VBM, leading to the increasing absorption tail of the L-PCN-1.0 in Figs. 5a and S20. Interestingly, we note that the $\mathrm{P}$ doping can spatially separate the highest occupied molecular orbital (HOMO) and lowest unoccupied 

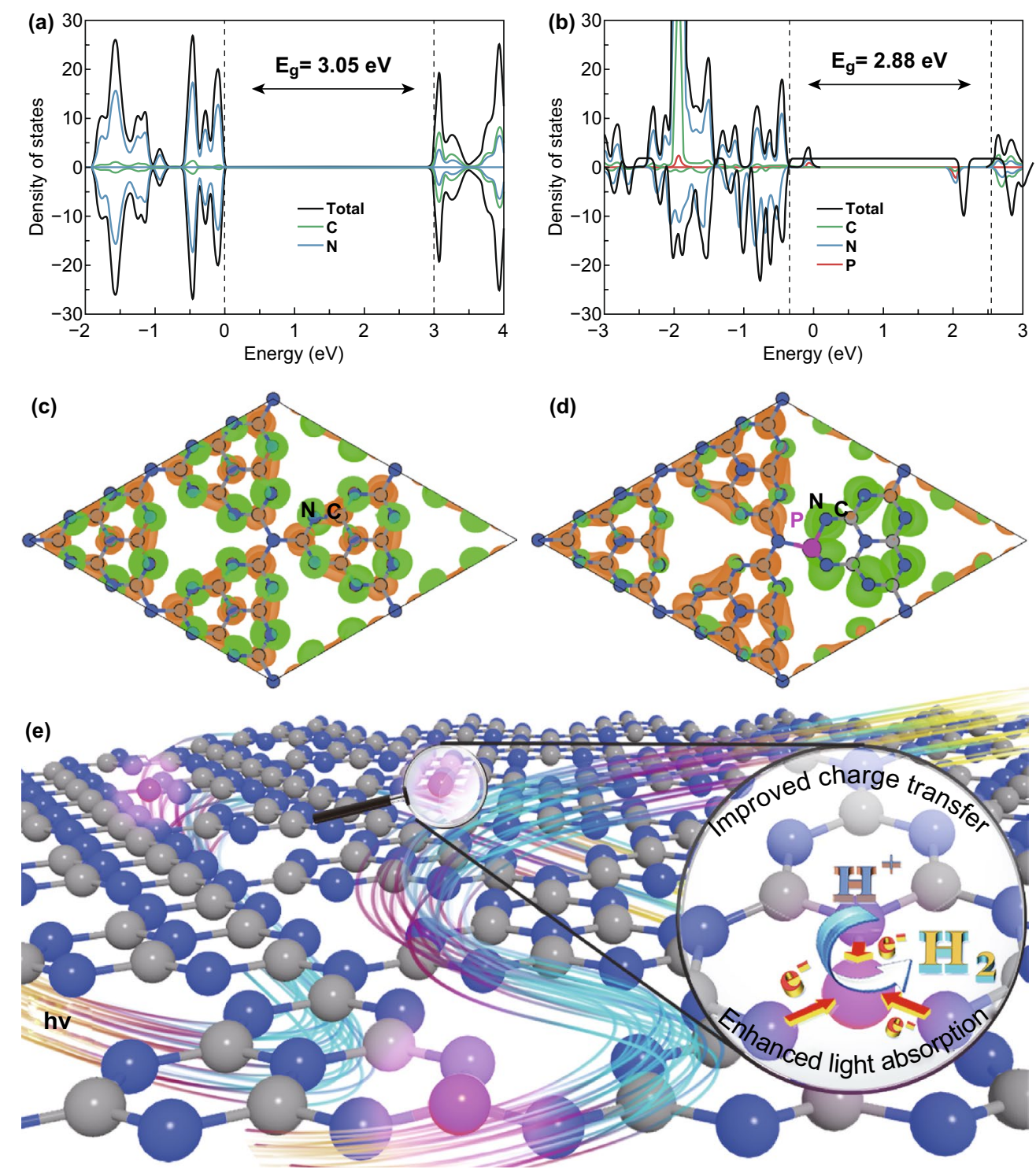

Fig. 8 DFT calculations and photocatalytic mechanism of L-PCN. Total density of states and partial density of states for a CN and b P-doped $\mathrm{CN}$. The electron density distributions of the highest occupied (green) and lowest unoccupied orbit (orange) of $\mathbf{c} \mathrm{CN}$ and $\mathbf{d}$ P-doped $\mathrm{CN}$ with an isovalue of $0.02 e \AA^{-3}$. e Illustration of the photocatalytic process over L-PCN photocatalyst for hydrogen evolution. Gray, blue, and pink spheres represent the $\mathrm{C}, \mathrm{N}$, and $\mathrm{P}$ atoms. (Color figure online)

molecular orbital (LUMO). For the pristine CN, the HOMO and LUMO are mainly consisted of $\mathrm{N}$ and $\mathrm{C}$ orbital, respectively, and distribute on the heptazine units evenly (Fig. 8c). By contrast, the HOMO of P-doped mainly distributes on the heptazine doped with $\mathrm{P}$ atom, while the LUMO distributes on other heptazines, as shown in Fig. 8d. It results in an efficient spatial separation of photogenerated carriers, thereby inhibiting their recombination.

Based on the above discussion, it is conceivable that the remarkable photo-/electrocatalytic water splitting activity of the L-PCN-1.0 catalyst can be ascribed to the following factors. The modification of the electronic structure owing to $\mathrm{P}$ doping effect promotes more visible light harvesting and provides efficient charge transfer channels between different tri-s-triazine rings (Fig. 8e). Based on a combined analysis of DRS and the Mott-Schottky plots (Figs. 5a, 6a, b), L-PCN-1.0 can absorb more visible light and thus have a higher flat potential and donor density, which promotes the photocatalytic reaction thermodynamically. Additionally, the unique hierarchical self-assembly of well-defined louver-like 
P-doped CN nanowire arrays exhibits a larger specific surface area $\left(121 \mathrm{~m}^{2} \mathrm{~g}^{-1}\right.$, Fig. 3a), more boundaries, and active sites exposed, as well as higher mass transport in hydrogen evolution process originated from the clearance spaces among $\mathrm{CN}$ nanowires (SEM image, Fig. 2b), resulting in excellent hydrogen production performance. Furthermore, L-PCN-1.0 possesses prolonged separation life of photogenerated carriers. The sharp decreased PL spectra (Fig. 5c), the lengthened decay time of charge carriers (Fig. 5d), smaller diameter of the EIS Nyquist curve, and stronger response of the transient photocurrent plots (Fig. 6c, d) all revealed the markedly improved electromobility efficiency of charge carriers; these diversified active elements pave the way for highly synergistic improving the hydrogen evolution reaction under visible light irradiation.

\section{Conclusions}

In summary, we show a facile yet effective route toward precisely manipulated synthesis of well-defined louver-like P-doped carbon nitride nanowire arrays via a hierarchical supramolecular self-assembly method by regulating the hydrogen bond. The controlled realization of self-assembly P-doped carbon nitride nanowire arrays with appropriate separation and spatial location of each $\mathrm{CN}$ nanowire integrates full potential of assembled nanostructures by virtue of the collective properties of single $\mathrm{CN}$ nanowire as well as ultrastability owing to the well-defined frame. Owing to the unique louver-like nanostructure with optimized optical and electronic structure characters, L-PCN nanowire arrays demonstrate its efficient and hyperstatic visible light-driven water splitting hydrogen evolution activity of $1872.9 \mu \mathrm{mol} \mathrm{h}^{-1} \mathrm{~g}^{-1}$ and an apparent quantum efficiency of $6.93 \%$ at $420 \pm 15 \mathrm{~nm}$. This hierarchical self-assembly approach releases the potential of single nanostructures selfassembly into diversified nanostructure arrays and facilitates the manufacturing of nanomaterials with diverse shapes and functional properties.

Acknowledgements The authors are grateful to the National Natural Science Foundation of China (Nos. 51772085 and U1830138) and Hunan Provincial Innovation Foundation for Postgraduate (No. CX20190311).

Open Access This article is licensed under a Creative Commons Attribution 4.0 International License, which permits use, sharing, adaptation, distribution and reproduction in any medium or format, as long as you give appropriate credit to the original author(s) and the source, provide a link to the Creative Commons licence, and indicate if changes were made. The images or other third party material in this article are included in the article's Creative Commons licence, unless indicated otherwise in a credit line to the material. If material is not included in the article's Creative Commons licence and your intended use is not permitted by statutory regulation or exceeds the permitted use, you will need to obtain permission directly from the copyright holder. To view a copy of this licence, visit http://creativecommons.org/licenses/by/4.0/.

Electronic supplementary material The online version of this article (https://doi.org/10.1007/s40820-020-0399-1) contains supplementary material, which is available to authorized users.

\section{References}

1. T. Aida, E.W. Meijer, S.I. Stupp, Functional supramolecular polymers. Science 335(6070), 813-817 (2012). https://doi. org/10.1126/science. 1205962

2. X. Wang, P.Y. Gao, Y.Y. Yang, H.X. Guo, D.C. Wu, Dynamic and programmable morphology and size evolution via a living hierarchical self-assembly strategy. Nat. Commun. 9, 2772 (2018). https://doi.org/10.1038/s41467-018-05142-3

3. L. Adler-Abramovich, E. Gazit, The physical properties of supramolecular peptide assemblies: from building block association to technological applications. Chem. Soc. Rev. 43(20), 6881-6893 (2014). https://doi.org/10.1039/c4cs00164h

4. M.G. Kibria, F.A. Chowdhury, S. Zhao, B. AlOtaibi, M.L. Trudeau, H. Guo, Z. Mi, Visible light-driven efficient overall water splitting using p-type metal-nitride nanowire arrays. Nat. Commun. 6, 8 (2015). https://doi.org/10.1038/ncomm s7797

5. L. Lv, Z.X. Yang, K. Chen, C.D. Wang, Y.J. Xiong, 2D layered double hydroxides for oxygen evolution reaction: from fundamental design to application. Adv. Energy Mater. 9(17), 1803358 (2019). https://doi.org/10.1002/aenm.201803358

6. Y. Zhang, Q. Liao, X.G. Wang, J.N.A. Yao, H.B. Fu, Lattice-matched epitaxial growth of organic heterostructures for integrated optoelectronic application. Angew. Chem. Int. Ed. 56(13), 3616-3620 (2017). https://doi.org/10.1002/anie.20170 0447

7. J. Yao, H. Yan, C.M. Lieber, A nanoscale combing technique for the large-scale assembly of highly aligned nanowires. Nat. Nanotechnol. 8(5), 329-335 (2013). https://doi.org/10.1038/ nnano.2013.55

8. M.P. Zhuo, J.J. Wu, X.D. Wang, Y.C. Tao, Y. Yuan, L.S. Liao, Hierarchical self-assembly of organic heterostructure nanowires. Nat. Commun. 10, 9 (2019). https://doi.org/10.1038/ s41467-019-11731-7

9. S. Kawasaki, R. Takahashi, T. Yamamoto, M. Kobayashi, H. Kumigashira et al., Photoelectrochemical water splitting enhanced by self-assembled metal nanopillars embedded in an oxide semiconductor photoelectrode. Nat. Commun. 7, 6 (2016). https://doi.org/10.1038/ncomms11818 
10. S. Guo, Z. Deng, M. Li, B. Jiang, C. Tian, Q. Pan, H. Fu, Phosphorus-doped carbon nitride tubes with a layered micro-nanostructure for enhanced visible-light photocatalytic hydrogen evolution. Angew. Chem. Int. Ed. 55(5), 1830-1834 (2016). https://doi.org/10.1002/ange.201508505

11. H.L. Sun, Y. Chen, J. Zhao, Y. Liu, Photocontrolled reversible conversion of nanotube and nanoparticle mediated by -cyclodextrin dimers. Angew. Chem. Int. Ed. 54(32), 93769380 (2015). https://doi.org/10.1002/anie.201503614

12. Y. Kim, W. Li, S. Shin, M. Lee, Development of toroidal nanostructures by self-assembly: rational designs and applications. Acc. Chem. Res. 46(12), 2888-2897 (2013). https ://doi.org/10.1021/ar400027c

13. H.C. Sun, J.G. Li, L. Lv, Z.S. Li, X. Ao et al., Engineering hierarchical $\mathrm{CoSe} / \mathrm{NiFe}$ layered-double-hydroxide nanoarrays as high efficient bifunctional electrocatalyst for overall water splitting. J. Power Sources 425, 138-146 (2019). https ://doi.org/10.1016/j.jpowsour.2019.04.014

14. B. Zhang, F.Z. Wang, C.Q. Zhu, Q. Li, J.N. Song, M.J. Zheng, L. Ma, W.Z. Shen, A facile self-assembly synthesis of hexagonal Zno nanosheet films and their photoelectrochemical properties. Nano-Micro Lett. 8(2), 137-142 (2016). https://doi.org/10.1007/s40820-015-0068-y

15. S.P. Wang, W. Lin, X.L. Wang, T.Y. Cen, H.J. Xie et al., Controllable hierarchical self-assembly of porphyrin-derived supra-amphiphiles. Nat. Commun. 10, 12 (2019). https://doi. org/10.1038/s41467-019-09363-y

16. S. Kunwar, M. Sui, Q. Zhang, P. Pandey, M.Y. Li, J. Lee, Various silver nanostructures on sapphire using plasmon self-assembly and dewetting of thin films. Nano-Micro Lett. 9(2), 17 (2017). https://doi.org/10.1007/s40820-016-0120-6

17. X. Wang, K. Maeda, A. Thomas, K. Takanabe, G. Xin, J.M. Carlsson, K. Domen, M. Antonietti, A metal-free polymeric photocatalyst for hydrogen production from water under visible light. Nat. Mater. 8(1), 76-80 (2009). https://doi. org/10.1038/NMAT2317

18. W. Wu, Z. Ruan, J. Li, Y. Li, Y. Jiang et al., In situ preparation and analysis of bimetal Co-doped mesoporous graphitic carbon nitride with enhanced photocatalytic activity. NanoMicro Lett. 11, 10 (2019). https://doi.org/10.1007/s4082 0-018-0236-y

19. Y.R. Li, T.T. Kong, S.H. Shen, Artificial photosynthesis with polymeric carbon nitride: when meeting metal nanoparticles, single atoms, and molecular complexes. Small 15(32), 1900772 (2019). https://doi.org/10.1002/smll.201900772

20. K. Xiao, L. Chen, R.T. Chen, T. Heil, S.D.C. Lemus et al., Artificial light-driven ion pump for photoelectric energy conversion. Nat. Commun. 10, 74 (2019). https://doi. org/10.1038/s41467-018-08029-5

21. S. Cao, J. Low, J. Yu, M. Jaroniec, Polymeric photocatalysts based on graphitic carbon nitride. Adv. Mater. 27(13), 2150-2176 (2015). https://doi.org/10.1002/adma.201500033

22. Y.J. Fu, C.A. Liu, M.L. Zhang, C. Zhu, H. Li et al., Photocatalytic $\mathrm{H}_{2} \mathrm{O}_{2}$ and $\mathrm{H}_{2}$ generation from living chlorella vulgaris and carbon micro particle comodified $g-\mathrm{C}_{3} \mathrm{~N}_{4}$.
Adv. Energy Mater. 8(34), 1802525 (2018). https://doi. org/10.1002/aenm.201802525

23. B. Li, Y. Si, B.X. Zhou, Q. Fang, Y.Y. Li et al., Dopinginduced hydrogen-bond engineering in polymeric carbon nitride to significantly boost the photocatalytic $\mathrm{H}_{2}$ evolution performance. ACS Appl. Mater. Interfaces 11(19), 1734117349 (2019). https://doi.org/10.1021/acsami.8b22366

24. S. Yang, Y. Gong, J. Zhang, L. Zhan, L. Ma et al., Exfoliated graphitic carbon nitride nanosheets as efficient catalysts for hydrogen evolution under visible light. Adv. Mater. 25(17), 2452-2456 (2013). https://doi.org/10.1002/adma.201204453

25. Y. Kang, Y. Yang, L.C. Yin, X. Kang, L. Wang, G. Liu, H.M. Cheng, Selective breaking of hydrogen bonds of layered carbon nitride for visible light photocatalysis. Adv. Mater. 28(30), 6471-6477 (2016). https://doi.org/10.1002/ adma.201601567

26. Z. Lin, X. Wang, Nanostructure engineering and doping of conjugated carbon nitride semiconductors for hydrogen photosynthesis. Angew. Chem. Int. Ed. 25(6), 1779-1782 (2013). https://doi.org/10.1002/anie.201209017

27. Y. Zhou, L. Zhang, J. Liu, X. Fan, B. Wang et al., Brand new P-doped $g-\mathrm{C}_{3} \mathrm{~N}_{4}$ : enhanced photocatalytic activity for $\mathrm{H}_{2}$ evolution and Rhodamine $\mathrm{B}$ degradation under visible light. J. Mater. Chem. A 3(7), 3862-3867 (2015). https://doi. org/10.1039/c4ta05292g

28. Y. Zhang, T. Mori, J. Ye, M. Antonietti, Phosphorus-doped carbon nitride solid: enhanced electrical conductivity and photocurrent generation. J. Am. Chem. Soc. 132(18), 6294-6295 (2010). https://doi.org/10.1021/ja101749y

29. R.J. Ran, T.Y. Ma, G. Gao, X. Du, S. Qiao, Porous P-doped graphitic carbon nitride nanosheets for synergistically enhanced visible-light photocatalytic $\mathrm{H}_{2}$ production. Energy Environ. Sci. 8(12), 3708-3717 (2015). https://doi. org/10.1039/c5ee02650d

30. Q.X. Liu, C.M. Zeng, Z.H. Xie, L.H. Ai, Y.Y. Liu et al., Cobalt@ nitrogen-doped bamboo-structured carbon nanotube to boost photocatalytic hydrogen evolution on carbon nitride. Appl. Catal. B Environ. 254, 443-451 (2019). https://doi. org/10.1016/j.apcatb.2019.04.098

31. K. Liu, Y.T. Kang, Z.Q. Wang, X. Zhang, 25th anniversary article: reversible and adaptive functional supramolecular materials: "Noncovalent interaction" matters. Adv. Mater. 25(39), 5530-5548 (2013). https://doi.org/10.1002/adma2 01302015

32. M. Shalom, S. Inal, C. Fettkenhauer, D. Neher, M. Antonietti, Improving carbon nitride photocatalysis by supramolecular preorganization of monomers. J. Am. Chem. Soc. 135(19), 7118-7121 (2013). https://doi.org/10.1021/ja402521s

33. G. Ge, X.W. Guo, C.S. Song, Z.K. Zhao, Reconstructing supramolecular aggregates to nitrogen-deficient $\mathrm{g}-\mathrm{C}_{3} \mathrm{~N}_{4}$ bunchy tubes with enhanced photocatalysis for $\mathrm{H}_{2}$ production. ACS Appl. Mater. Interfaces 10(22), 18746-18753 (2018). https://doi.org/10.1021/acsami.8b04227

34. Z.W. Tong, D. Yang, Y.Y. Sun, Y.H. Nan, Z.Y. Jiang, Tubular $\mathrm{g}-\mathrm{C}_{3} \mathrm{~N}_{4}$ isotype heterojunction: enhanced visible-light 
photocatalytic activity through cooperative manipulation of oriented electron and hole transfer. Small 12(30), 4093-4101 (2016). https://doi.org/10.1002/smll.201601660

35. N.A. Wasio, R.C. Quardokus, R.P. Forrest, C.S. Lent, S.A. Corcelli, J.A. Christie, K.W. Henderson, S.A. Kandel, Selfassembly of hydrogen-bonded two-dimensional quasicrystals. Nature 507(7490), 86-89 (2014). https://doi.org/10.1038/natur e12993

36. M. Shalom, M. Guttentag, C. Fettkenhauer, S. Inal, D. Neher, A. Llobet, M. Antonietti, In situ formation of heterojunctions in modified graphitic carbon nitride: synthesis and noble metal free photocatalysis. Chem. Mater. 26(19), 5812-5818 (2014). https://doi.org/10.1021/cm503258z

37. Y.Y. Li, Y. Si, B.X. Zhou, W.Q. Huang, W.Y. Hu, A.L. Pan, X.X. Fan, G.F. Huang, Strategy to boost catalytic activity of polymeric carbon nitride: synergistic effect of controllable in situ surface engineering and morphology. Nanoscale 11(35), 16393-16405 (2019). https://doi.org/10.1039/c9nr0 $5413 \mathrm{~h}$

38. B.X. Zhou, S.S. Ding, B.J. Zhang, L. Xu, R.S. Chen et al., Dimensional transformation and morphological control of graphitic carbon nitride from water-based supramolecular assembly for photocatalytic hydrogen evolution: from 3D to 2D and 1D nanostructures. Appl. Catal. B Environ. 254, 321-328 (2019). https://doi.org/10.1016/j.apcatb.2019.05.015

39. Y. Ishida, L. Chabanne, M. Antonietti, M. Shalom, Morphology control and photocatalysis enhancement by the one-pot synthesis of carbon nitride from preorganized hydrogenbonded supramolecular precursors. Langmuir 30(2), 447-451 (2014). https://doi.org/10.1021/la404101h

40. L.S. Zhang, N. Ding, M. Hashimoto, K. Iwasaki, N. Chikamori et al., Sodium-doped carbon nitride nanotubes for efficient visible light-driven hydrogen production. Nano Res. 11(4), 22952309 (2018). https://doi.org/10.1007/s12274-017-1853-3

41. Y.T. Gao, F. Hou, S. Hu, B.G. Wu, Y. Wang, H.Q. Zhang, B.J. Jiang, H.G. Fu, Graphene quantum-dot-modified hexagonal tubular carbon nitride for visible-light photocatalytic hydrogen evolution. Chemcatchem 10(6), 1330-1335 (2018). https://doi. org/10.1002/cctc. 201701823

42. B. Liu, L.Q. Ye, R. Wang, J.F. Yang, Y.X. Zhang, R. Guan, L.H. Tian, X.B. Chen, Phosphorus-doped graphitic carbon nitride nanotubes with amino-rich surface for efficient $\mathrm{CO}_{2}$ capture, enhanced photocatalytic activity, and product selectivity. ACS Appl. Mater. Interfaces 10(4), 4001-4009 (2018). https://doi.org/10.1021/acsami.7b17503

43. G. Kresse, J. Furthmuller, Efficiency of ab initio total energy calculations for metals and semiconductors using a plane-wave basis set. Comput. Mater. Sci. 6(1), 15-50 (1996). https://doi. org/10.1016/0927-0256(96)00008-0

44. G. Kresse, J. Furthmuller, Efficient iterative schemes for ab initio total-energy calculations using a plane-wave basis set. Phys. Rev. B Condens. Matter 54(16), 11169-11186 (1996). https://doi.org/10.1103/PhysRevB.54.11169

45. J.P. Perdew, K. Burke, M. Ernzerhof, Generalized gradient approximation made simple. Phys. Rev. Lett. 77(18), 38653868 (1996). https://doi.org/10.1103/PhysRevLett.77.3865
46. S. Grimme, Semiempirical GGA-type density functional constructed with a long-range dispersion correction. J. Comput. Chem. 27(15), 1787-1799 (2006). https://doi.org/10.1002/ jcc. 20495

47. J. Heyd, G.E. Scuseria, M. Ernzerhof, Hybrid functionals based on a screened coulomb potential. J. Chem. Phys. 118(18), 8207-8215 (2003). https://doi.org/10.1063/1.15640 60

48. J. Paier, M. Marsman, K. Hummer, G. Kresse, I.C. Gerber, J.G. Angyan, Screened hybrid density functionals applied to solids. J. Chem. Phys. 124(15), 154709 (2006). https://doi. org/10.1063/1.2187006

49. Q. Liang, Z. Li, Z.H. Huang, F. Kang, Q.H. Yang, Holey graphitic carbon nitride nanosheets with carbon vacancies for highly improved photocatalytic hydrogen production. Adv. Funct. Mater. 25(44), 6885-6892 (2016). https://doi. org/10.1002/adfm.201503221

50. Y.F. Li, R.X. Jin, Y. Xing, J.Q. Li, S.Y. Song, X.C. Liu, M. $\mathrm{Li}$, R.C. Jin, Macroscopic foam-like holey ultrathin $\mathrm{g}-\mathrm{C}_{3} \mathrm{~N}_{4}$ nanosheets for drastic improvement of visible-light photocatalytic activity. Adv. Energy Mater. 6(24), 5 (2016). https ://doi.org/10.1002/aenm.201601273

51. X. Ao, W. Zhang, Z.S. Li, J.G. Li, L. Soule et al., Markedly enhanced oxygen reduction activity of single-atom $\mathrm{Fe}$ catalysts via integration with Fe nanoclusters. ACS Nano 13(10), 11853-11862 (2019). https://doi.org/10.1021/acsna no. 9 b05913

52. Y.C. Bao, K.Z. Chen, $\mathrm{AgCl} / \mathrm{Ag} / \mathrm{g}-\mathrm{C}_{3} \mathrm{~N}_{4}$ hybrid composites: preparation, visible light-driven photocatalytic activity and mechanism. Nano-Micro Lett. 8(2), 182-192 (2016). https ://doi.org/10.1007/s40820-015-0076-y

53. F. Raziq, Y. Qu, M. Humayun, A. Zada, H.T. Yu, L.Q. Jing, Synthesis of $\mathrm{SnO}_{2} / \mathrm{B}-\mathrm{P}$ codoped $\mathrm{g}-\mathrm{C}_{3} \mathrm{~N}_{4}$ nanocomposites as efficient cocatalyst-free visible-light photocatalysts for $\mathrm{CO}_{2}$ conversion and pollutant degradation. Appl. Catal. B Environ. 201, 486-494 (2017). https://doi.org/10.1016/j.apcat b. 2016.08 .057

54. S. Zhang, S. Song, P.C. Gu, R. Ma, D.L. Wei et al., Visiblelight-driven activation of persulfate over cyano and hydroxyl group co-modified mesoporous $\mathrm{g}-\mathrm{C}_{3} \mathrm{~N}_{4}$ for boosting bisphenol a degradation. J. Mater. Chem. A 7(10), 5552-5560 (2019). https://doi.org/10.1039/c9ta00339h

55. J. Zhang, M. Zhang, G. Zhang, X. Wang, Synthesis of carbon nitride semiconductors in sulfur flux for water photoredox catalysis. ACS Catal. 2(2), 940-948 (2012). https://doi. org/10.1021/cs300167b

56. H. Yu, R. Shi, Y. Zhao, T. Bian, Y. Zhao et al., Alkaliassisted synthesis of nitrogen deficient graphitic carbon nitride with tunable band structures for efficient visiblelight-driven hydrogen evolution. Adv. Mater. 29(16), 1605148 (2017). https://doi.org/10.1002/adma.201605148

57. C.H. Liu, F. Wang, J. Zhang, K. Wang, Y.Y. Qiu, Q. Liang, Z.D. Chen, Efficient photoelectrochemical water splitting by $\mathrm{g}-\mathrm{C}_{3} \mathrm{~N}_{4} / \mathrm{TiO}_{2}$ nanotube array heterostructures. NanoMicro Lett. 10(2), 37 (2018). https://doi.org/10.1007/s4082 0-018-0192-6 
58. K. Li, W.D. Zhang, Creating graphitic carbon nitride based donor- $\pi$-acceptor- $\pi$-donor structured catalysts for highly photocatalytic hydrogen evolution. Small 14(12), 1703599 (2018). https://doi.org/10.1002/smll.201703599

59. Y.P. Zhu, T.Z. Ren, Z.Y. Yuana, Mesoporous phosphorusdoped $g-\mathrm{C}_{3} \mathrm{~N}_{4}$ nanostructured flowers with superior photocatalytic hydrogen evolution performance. ACS Appl. Mater. Interfaces 7(30), 16850-16856 (2015). https://doi. org/10.1021/acsami.5b04947

60. S.E. Guo, Y.Q. Tang, Y. Xie, C.G. Tian, Q.M. Feng, W. Zhou, B.J. Jiang, P-doped tubular $\mathrm{g}-\mathrm{C}_{3} \mathrm{~N}_{4}$ with surface carbon defects: universal synthesis and enhanced visible-light photocatalytic hydrogen production. Appl. Catal. B Environ. 218, 664-671 (2017). https://doi.org/10.1016/j.apcat b.2017.07.022

61. M. Wu, J. Zhang, B.B. He, H.W. Wang, R. Wang, Y.S. Gong, In-situ construction of coral-like porous P-doped g- $\mathrm{C}_{3} \mathrm{~N}_{4}$ tubes with hybrid 1D/2D architecture and high efficient photocatalytic hydrogen evolution. Appl. Catal. B Environ. 241, 159-166 (2019). https://doi.org/10.1016/j.apcatb.2018.09.037

62. J. Liu, Y. Liu, N. Liu, Y. Han, X. Zhang et al., Metal-free efficient photocatalyst for stable visible water splitting via a two-electron pathway. Science 46(23), 970-974 (2015). https ://doi.org/10.1126/science.aaa3145
63. Z.Y. Zhang, J.D. Huang, M.Y. Zhang, L. Yuan, B. Dong, Ultrathin hexagonal $\mathrm{SnS}$, nanosheets coupled with $\mathrm{g}-\mathrm{C}_{3} \mathrm{~N}_{4}$ nanosheets as $2 \mathrm{D} / 2 \mathrm{D}$ heterojunction photocatalysts toward high photocatalytic activity. Appl. Catal. B Environ. 163, 298-305 (2015). https://doi.org/10.1016/j.apcat b.2014.08.013

64. W. Xing, C. Li, G. Chen, Z. Han, Y. Zhou, Y. Hu, Q. Meng, Incorporating a novel metal-free interlayer into g- $\mathrm{C}_{3} \mathrm{~N}_{4}$ framework for efficiency enhanced photocatalytic $\mathrm{H}_{2}$ evolution activity. Appl. Catal. B Environ. 203, 65-71 (2017). https:// doi.org/10.1016/j.apcatb.2016.09.075

65. S. Chu, Y. Wang, Y. Guo, J.Y. Feng, C.C. Wang, W.J. Luo, X.X. Fan, Z.G. Zou, Band structure engineering of carbon nitride: in search of a polymer photocatalyst with high photooxidation property. ACS Catal. 3(5), 912-919 (2013). https ://doi.org/10.1021/cs4000624

66. J.G. Li, Y. Gu, H.C. Sun, L. Lv, Z.S. Li et al., Engineering the coupling interface of rhombic dodecahedral NiCoP/C@FeOOH nanocages toward enhanced water oxidation. Nanoscale 11(42), 19959-19968 (2019). https://doi. org/10.1039/c9nr07967j 